\title{
Dynamical properties of fish schooling behavior
}

\author{
MASAAKI ISHIKAWA \\ Department of Civil and Environmental Engineering, Kisarazu National College of Technology, 2-11-1 Kiyomidai-Higashi \\ Kisarazu, Chiba 292-0041, Japan (ishikawa@wangan.c.kisarazuac.jp)
}

\section{KEYWORDS: fish schooling behavior, power spectrum analysis, individual based model, Japanese dace}

\section{INTRODUCTION}

In this article we describe an experimental study on the dynamics of fish schooling behaviors It is common knowledge that fish schooling behaviors are complex phenomena. The grain of wood, hum of an insect, traffic of expressway and pulsatory motion, in all respects of natural phenomena, $1 / f$ fluctuation is confirmed.

On the hypothesis that natural phenomena consist with simple basic rules and randomness, we tried to develop fish schooling behaviors model and software for simulation. To construct model of fish schooling behaviors, we are able to design optimum entrance location of fishway and effective shape of escape zone for fish on flood, and so forth.

\section{MATERIALS AND METHODS}

At first, in order to obtain information on dynamical fish school behaviors, experiments with Japanese dace Tribolodon hakonensis ware carried out. The behaviors of Japanese dace in both static and flowing water area were recorded using a digital video camera, which was fixed on a ceiling board and covered the whole observation water area. The observation water area was a part of experimental prismatic channel. Its size was a $0.80 \mathrm{~m}$ width and $1.80 \mathrm{~m}$ length in rectangular shape. In the upper and lower observation water area, we set up fish screen net for protection against fish moving out of area. At intervals one second, image were captured from digital videotapes. Coordinates data of each fish head position was digitized from image data. Water depth of observation area was $0.15 \mathrm{~m}$ in both cases. In case of flowing water, discharge was $0.012 \mathrm{~m}^{3} / \mathrm{s}$ and flow speed in $0.10 \mathrm{~m}$ mesh grid points were measured by three dimensional electromagnetic current meter. Average body length of test fishes was about $0.10 \mathrm{~m}$.

Individual based model (IBM) was proposed by Creg W.Reynolds ${ }^{2}$. IBM was method of making animated motion pictures. Wide view of flocks motion pattern was led from interaction among individuals. We referred to Reynolds's basic behavior rule for bird flocks

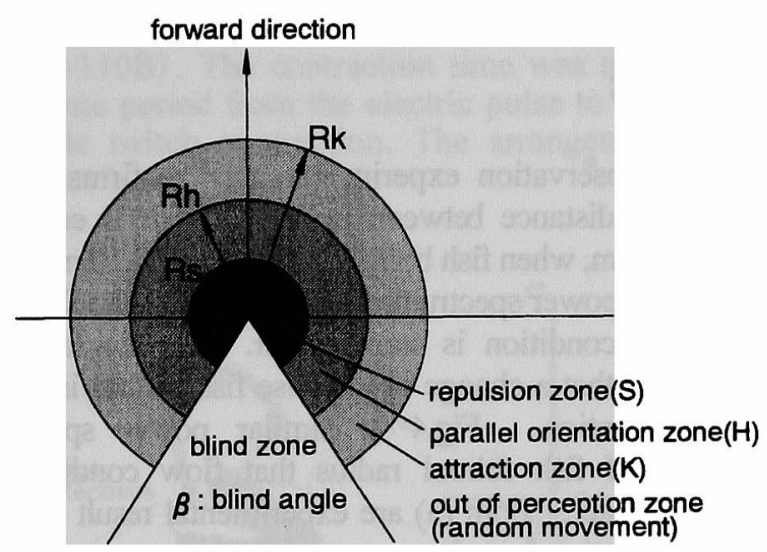

Fig.1 Basin behavior rule of fish for nearest other fish. The three response zone. Out of perception zone fish selected random movement.

and our observation experimental results. We set up basic behavior rule of model below.

Behavioral patterns of fish can be classified into repulsion, parallel orientation, attraction and random motion that correspond to distance from nearest individuals are shown in Fig.1. To the boundary, fish reflect on an angle of reflection that same as an angle of incidence. A matrix data of flowing speed distribution is input to the software. If swimming speed is grater than flowing speed, fish will swim against the flow, and swimming direction has a range with \pm 10 degrees. If swimming speed is smaller than flowing speed, fish seek the smallest speed zone from adjacent zone, and fish move to this zone in next step.

We defined fish school radius that indicate expanse of fish school. The definition is shown as Fig.2. The fish school radius $R f s$ is

$$
R f s=\sqrt{\sum_{i=1}^{N} r_{i}^{2} / N}
$$


and has unit of $\mathrm{m} ; N$ is the number of fish in the school; $r_{i}$ is the distance from gravity of school to each fish.

Time series data of fish school radius calculated from experimental and simulated observation coordinates data of each fish head position. Applied Fast Fourier Transform (FFT) Analysis to time series data of fish school radius, we got a relationship of power spectrum and frequency. In verification of individual based model, we compared computational results with experimental one, used power spectrum of fish school radius.

\section{RESULTS}

By fish observation experiments, this confirms that a minimum distance between individual fish is equal to about $0.03 \mathrm{~m}$, when fish body length is about $0.10 \mathrm{~m}$.

Fig. 3 is power spectrum diagram of fish school radius that flow condition is static water. This diagram has confirmed that a change of expanse fish school is $1 / \mathrm{f}$ or $1 / \mathrm{f}^{2}$ fluctuation. Fig.4 is similar power spectrum diagram of fish school radius that flow condition is flowing water. Both (a) are experimental result and (b) are computational result.

In both case static and flowing, fish school radius fluctuation experimental result has tendency of $1 / \mathrm{f}^{2}$ than computational result. If examined in detail experimental result, fish school radius fluctuation of static water case has monotonous tendency than flowing water case, but in computational result we don't confirm similar tendency clearly.

\section{DISCUSSION}

These results have confirmed the certain reliability of the model that applied concept of individual based model. Complex fish schooling behavior was expressed by simple basic behavior rules with randomness.

As in static water case, restriction to fish is smaller than flowing water case, fish behavior properties will be monotonous. In this study we investigated about fish response against flowing water. In order to improve precision of fish behavior model, investigation about fish response to substrates is needed.

This time, we developed two-dimensional model of fish school behavior. Reflection of influence pool and riffle requires three-dimensional model.

If fish school behavior is reappeared by computational model, we are able to estimate influence of river improvement to fish habitat before construction.

All the numerical computations were made by personal computer with Pentium2 processor.

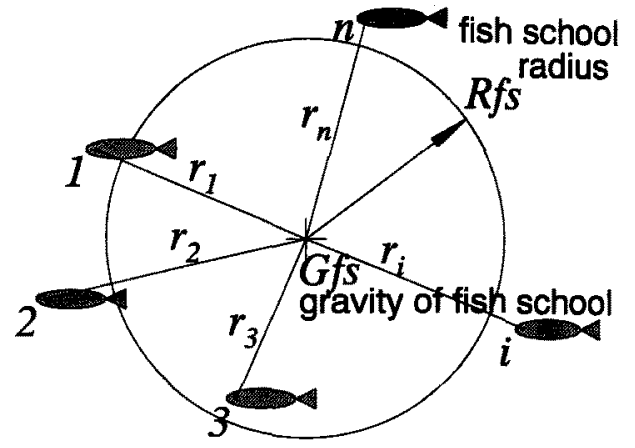

Fig.2 Definition of fish school radius $R f s$.
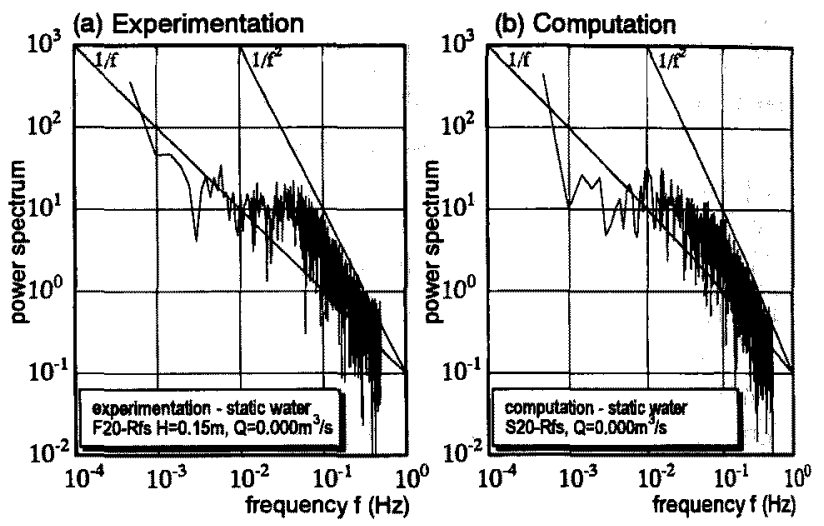

Fig 3 power spectrum diagram of static water.
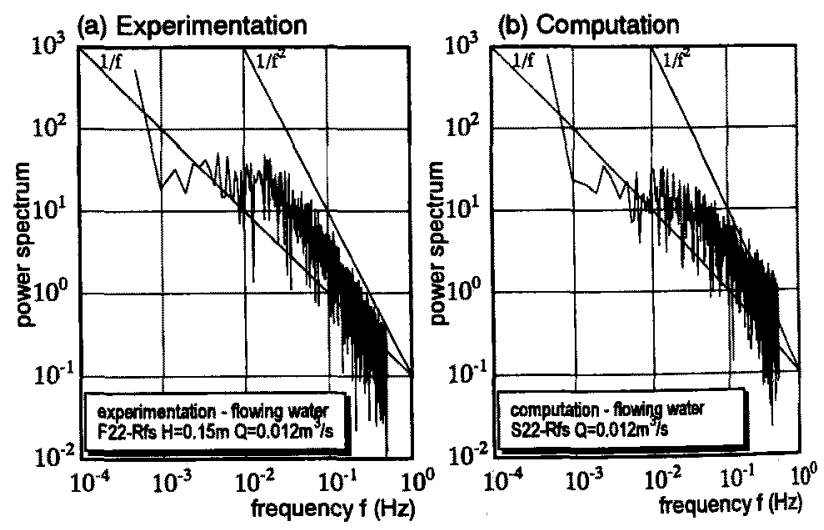

Fig.4 power spectrum diagram of flowing water.

\section{REFERENCES}

1. T.Musha: an idea of fluctuation, NHK Library 79 , NHK, 1998, [in Japanese].

2. Creg W.Reynolds: Flocks, Herds, and Schools, A Distributed Behavioral Model, The Proceeding of SIGGRAPH87, Vol.21(4), pp.25-34, 1987.

( http://chaos.kisarazu.ac.jp/ishikawa/English/VHS-Fish/ ) 